\title{
Real-time surveillance and response system for Ebola and other emerging infections
}

\author{
Pia D. MacDonald*, Gordon Cressman, Michael McKay, Stephen Loo, \\ Elizabeth McClure and Emily MacGuire
}

Center for Global Health, RTI International, Research Triangle Park, NC, USA

\section{Objective}

We will describe a real-time mobile surveillance and case management system designed to organize data collected by multiple officers about cases and their contacts. We will discuss this surveillance system and its application for Ebola and other infectious diseases in the Democratic Republic of the Congo (DRC) and other similar settings. We will review the technology, results, challenges, lessons-learned, and applicability to other contexts.

\section{Introduction}

Improving surveillance and response is a critical component of the Global Health Security Agenda. While it is impossible to predict where the next Ebola outbreak will occur, it is very likely that another outbreak will occur in the DRC. Of the 20 known outbreaks, 7 have occurred in the DRC, one as recently as 2014 . To rapidly detect and respond to an Ebola outbreak, we sought to develop a real-time surveillance and response system for use in DRC and similar settings. RTI International developed Coconut Surveillance mobile software, which is currently used for real-time malaria surveillance and response in Zanzibar, Africa, where malaria elimination efforts are underway. We took this system and adapted it for Ebola as a possible tool for surveillance and response to Ebola and other (re)emerging diseases. Plans include pilot testing functionality at clinical sites in DRC, where surveillance infrastructure is limited at the local level.

Coconut Surveillance is a mobile disease surveillance and rapid response system currently used for malaria elimination activities. It receives suspected positive case alerts from the field via mobile phones and uses mobile software to guide surveillance officers through a follow-up process. Coconut Surveillance runs on Android mobile devices that are used to coordinate work in the field as well as provide decision support during data collection and case management. In addition to standard case information, the GPS coordinates of the case's household are captured as well as malaria status of all household members. Data are collected and accessed off-line, and are synchronized with a shared database when Internet connectivity is available. This tool has been used successfully in Zanzibar for more than three years and has been recognized as one of the most advanced applications of its kind.

\section{Methods}

We adapted the Coconut Surveillance system for Ebola surveillance and response, and expanded the system for use with other communicable diseases. With a near real-time outbreak detection system for Ebola, we may reduce the response time and contain an outbreak faster. Using a cloud-based data repository, the modified Coconut System, known as Coconut Plus, also has the added value of case and case-contacts specific information sharing in real-time with the national, provincial, and district level public health authorities, who would have convenient and secure access to case and contact information via the Internet. The software modifications to the Coconut System have been informed by testing and stakeholder feedback.

\section{Results}

We have developed Coconut Plus around the Coconut software architecture, which allows the team to quickly develop specific workflows and applications, such as contact tracing, on top of a solid and well-supported base. Additionally, the adaptation was structured to accommodate the build-out of multiple diseases, and is uniquely helpful for diseases that require tracking many contacts. We were granted access in DRC to test interoperability with DHIS 2, the most widely used health information system software in Ebola effected countries. Coconut Plus is now using the DHIS 2 organizational hierarchy definition, which means that organizational hierarchy (including information on administrative units and health care facilities) can be exported directly from DHIS 2 to Coconut Plus. Stakeholder feedback on the usability and feasibility of the adapted system has been enthusiastic, and stressed the need for additional resources to make a pilot successful, including mobile phones and improved mobility of surveillance staff in the field. The following screencast provides an overview of the application: https://www. youtube.com/watch? $\mathrm{v}=\mathrm{jjLT} 3 \mathrm{pLLW}-\mathrm{U}$

\section{Conclusions}

Coconut Surveillance Plus solves an absence of a real-time mobile decision support disease surveillance and response system that can be used for Ebola and other infectious diseases in countries with limited surveillance infrastructure. More broadly, this system could also be used for many communicable diseases that require contact tracing and an urgent outbreak response in environments that require rapid scale up of a distributed surveillance, rapid response, and case management system.

\section{Keywords}

Surveillance system; Global Health Security; Informatics; Ebola; Malaria

\section{*Pia D. MacDonald}

E-mail: pmacdonald@rti.org 